\title{
MINIMUM SPOUTABLE GAS FLOW RATE IN SIDE-OUTLET SPOUTED BED WITH INNER DRAFT-TUBE
}

\author{
Hirotsugu HATTORI, KaZUYUKI TANAKA \\ AND KUNIHIKO TAKEDA \\ Department of Chemical Engineering, Shinshu University, \\ Ueda 386
}

\begin{abstract}
It has been found that the side-outlet spouted bed with inner draft-tube has the following advantageous characteristics stemming from the fact that simple rules hold at the beginning of spouting. When the gas velocity in the inner draft-tube increases and the ratio to terminal velocity of solid particles rises to a certain value, stable spouting begins. At the beginning of spouting the ratio of gas velocity in the gas inlet to terminal velocity of solid particles is also constant. These ratios do not change even if the size of the apparatus or the size of particles changes, as far as we have investigated on geometrically similar spouted beds. In addition, the gas streamlines are aproximately similar for geometrically similar spouted beds.

These characteristics of the side-outlet spouted bed with inner draft-tube facilitate the procedure for scale-up. For example, the minimum gas flow rate required for stable spouting can be easily predicted and the gas conversion can be easily estimated.
\end{abstract}

\section{Introduction}

In the side-outlet spouted bed with inner drafttube, the void fraction in the spout is approximately uniform throughout the height of the spout, in contrast to the case of the commonly used top-outlet spouted bed. In the spout region, the behaviour of the gas and solid particles is very similar to that in the pneumatic conveyer. The minimum spoutable gas velocity in the inner draft-tube must be equal to the minimum gas velocity in the pneumatic conveyer of the same void fraction. The minimum gas velocity in the inner draft-tube usually depends on the void fraction in the draft-tube. However, the void fractions in the inner draft-tube seem to be unchanged as far as we have investigated. So the minimum gas velocity in the inner draft-tube is unchanged and its ratio to terminal velocity of solid particles remains almost constant. The same rule holds when the size of the apparatus or the size of solid particles changes. These characteristics of the side-outlet spouted bed with inner draft-tube facilitate the procedure for scale-up.

In this study, air at room temperature and spherical glass particles from 1.8 to $0.27 \mathrm{~mm}$ in average diameter were used. Table 1 gives the physical properties of these particles.

Received February 2,1981. Correspondence concerning this article should be addressed to $\mathrm{H}$. Hattori.
1. Simple Rules Regarding Minimum Gas Velocities in Inner Draft-Tube and in Gas Inlet Required for Stable Spouting

\section{1 Minimum gas velocity in inner draft-tube}

When the gas velocity in the inner draft-tube rises to a few times the minimum fluidizing velocity, the solid particles begin to move vigorously up and down in the inner draft-tube. However, the gas velocity in the inner draft-tube is still smaller than the minimum spoutable one, and only a part of the solid particles sometimes moves to the annulus beyond the top of the inner draft-tube.

If the gas velocity increases sufficiently, pulsatory motion of solid particles vanishes and solid particles

Table 1 Physical properties of solid particles Glass beads (Specific gravity $=2.5$ )

\begin{tabular}{ccc} 
Tyler mesh & $\begin{array}{c}\text { Average diameter } \\
d_{p}[\mathrm{~mm}]\end{array}$ & $\begin{array}{c}\text { Terminal velocity } \\
U_{t}[\mathrm{~m} / \mathrm{sec}]\end{array}$ \\
\hline$-9+10$ & 1.8 & 10.5 \\
$-10+12$ & 1.5 & 9.4 \\
$-12+14$ & 1.3 & 8.5 \\
$-14+16$ & 1.1 & 7.4 \\
$-16+20$ & 0.91 & 6.5 \\
$-20+24$ & 0.77 & 5.6 \\
$-24+28$ & 0.65 & 4.8 \\
$-28+32$ & 0.54 & 4.1 \\
$-32+35$ & 0.46 & 3.5 \\
$-35+42$ & 0.38 & 2.9 \\
$-42+48$ & 0.32 & 2.4 \\
$-48+60$ & 0.27 & 2.0 \\
\hline
\end{tabular}


rise uniformly in the inner draft-tube. The rate of cyclic motion of solid particles increases and continuous cyclic motion sets in. Void fraction in the inner draft-tube becomes approximately uniform throughout the height of the draft-tube. At this time we will call the gas velocity in the inner draft-tube the minimum velocity required for stable spouting.

Figure 1 shows the minimum gas velocity in the inner draft-tube required for stable spouting. The ratio of bed depth $H$ to gas inlet diameter $D$ is 10 . It is clear that the minimum gas velocity in the inner drafttube is about 80 percent of the terminal velocity of solid particles for each size of solid particles. The gas velocity in the inner draft-tube was determined by dividing the volumetric flow rate of gas which passes through the inner draft-tube by the sectional area of the draft-tube. Terminal velocity of solid particles was calculated by the use of the relation between drag coefficient and Reynolds number of spherical particle. The relation between terminal velocities and the diameters of solid particles is shown in Table 1.

The minimum gas velocity in the inner draft-tube usually depends on the void fraction in the draft-tube. However, the minimum gas velocity does not change as far as we have investigated by the use of differentsized solid particles and different-sized apparatus. It is always about 80 percent of the terminal velocity of solid particles.

\section{2 Minimum gas velocities in inner draft-tube and in gas inlet}

Figure 2 illustrates the minimum gas velocity for stable spouting in the gas inlet and in the inner drafttube for the two side-outlet spouted beds. Between these two spouted beds, only the diameters of drafttube and therefore gas inlet are different, and the other dimensions of the apparatus are identical. The terminal velocity of solid particles is chosen as abscissa and the minimum gas velocities both in the inner drafttube and in the gas inlet as ordinate.

An approximately linear relationship exists between minimum gas velocity and the terminal velocity of solid particles in the inner draft-tube as well as in the gas inlet. The relation between the minimum gas velocity in the inner draft-tube and the terminal velocity of solid particles is correlated by a single straight line, and its slope is about 0.8 . This means that stable spouting sets in when the gas velocity in the inner draft-tube becomes about 80 percent of the terminal velocity of solid particles for these two spouted beds. On the other hand, the slopes of the lines for the gas inlet in Fig. 2 are slightly different from each other. In the side-outlet spouted bed with inner draft-tube, a part of the gas percolates through the annulus after it flows beyond the top of the inner draft-tube and the rest goes through the lower end of the draft-tube. The

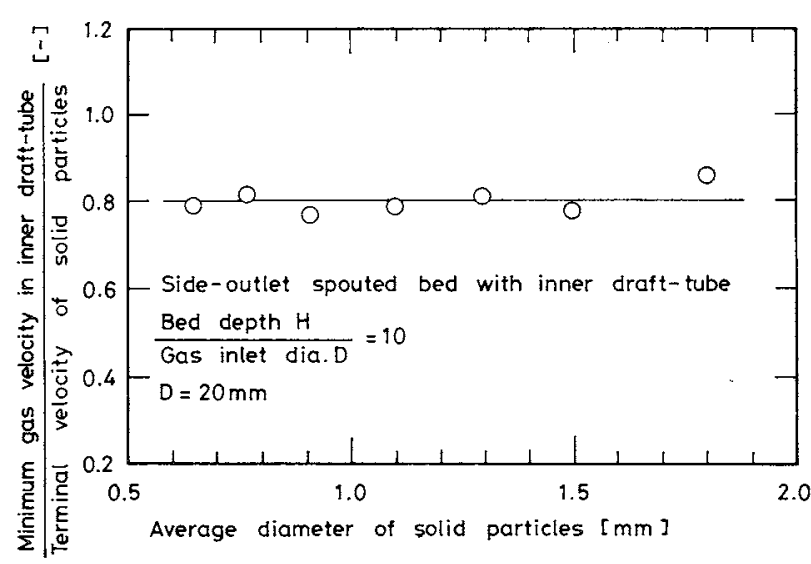

Fig. 1 Minimum gas velocity in inner draft-tube required for stable spouting for side-outlet spouted bed with inner draft-tube

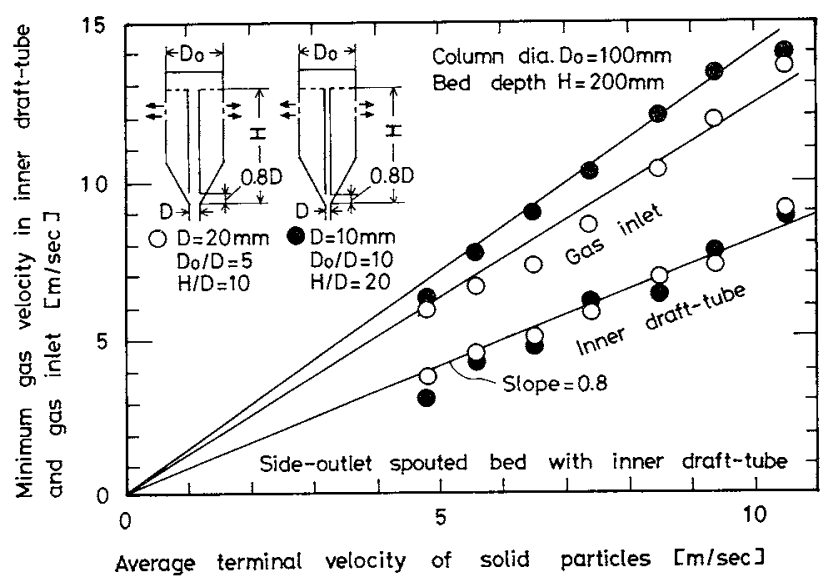

Fig. 2 Minimum gas velocities in inner draft tube and in gas inlet required for stable spouting

different flow ratio of the gas in these two parts gives different slopes in Fig. 2.

\section{Effect of Apparatus Dimensions}

The relation between the minimum gas velocity and the terminal velocity of solid particles in the inner draft-tube as well as in the gas inlet is studied for three spouted beds of geometrically similar shapes, as shown in Fig. 3. The ratios of the three sizes are 1, 1.5 and 2.0. The ratio of column diameter to gas inlet diameter is 5.0 , and the ratio of bed depth to gas inlet diameter is 10 for each size of spouted beds.

The results are shown in Fig. 4. As can be seen from Fig. 4, the minimum gas velocities both in the inner draft-tube and in the gas inlet show good agreement among the three sizes of spouted beds, and they are correlated by single straight lines. This result is caused by the fact that the gas streamlines in the annulus are approximately similar for these geometrically similar spouted beds, as shown in Fig. 3. When the shapes of the gas streamlines are geometrically similar, the ratio of the volumetric flow rate of the gas which passes through the inner draft-tube to the total 

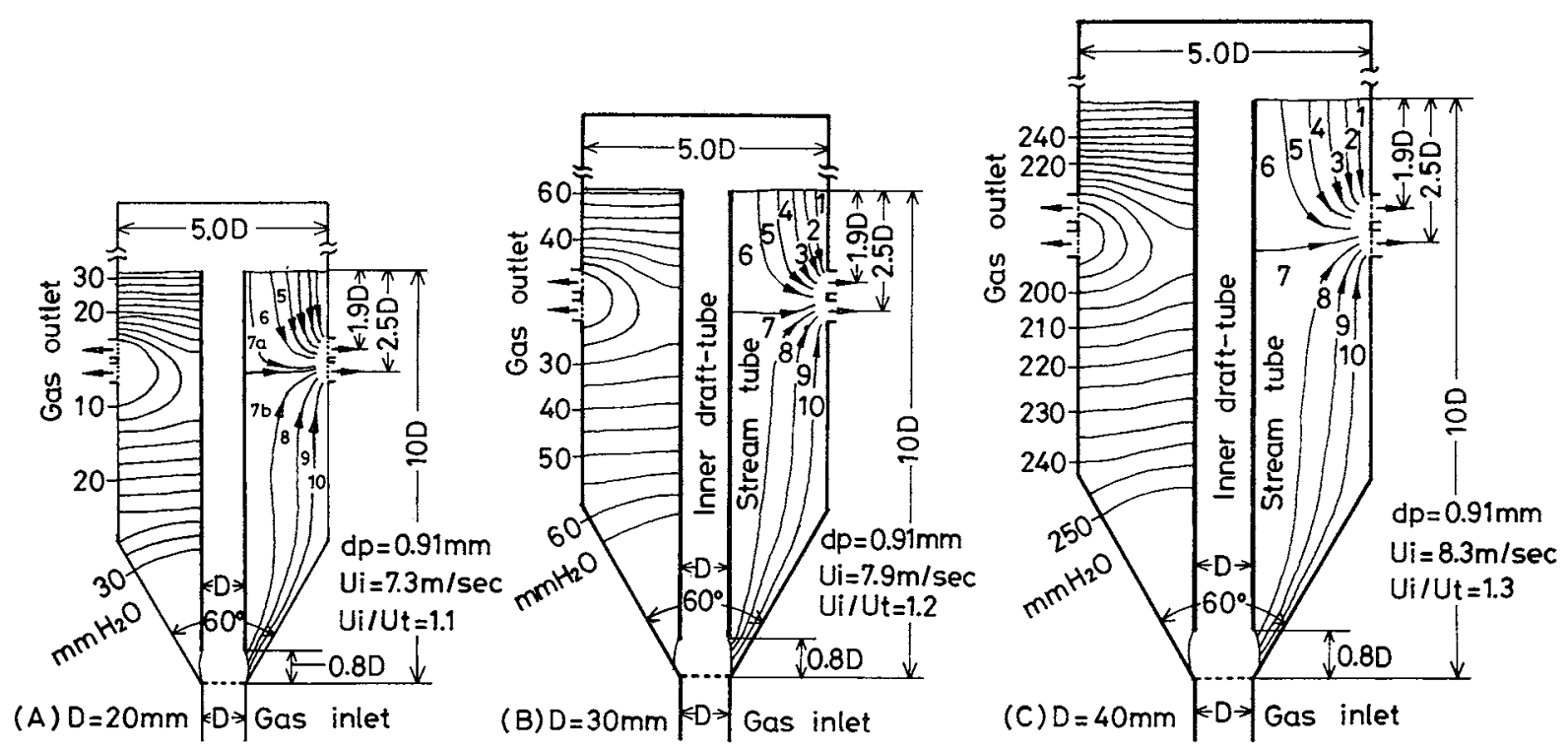

Fig 3 Pressure distribution and gas streamlines in annulus of similar-shaped side-outlet spouted beds with inner draft-tube

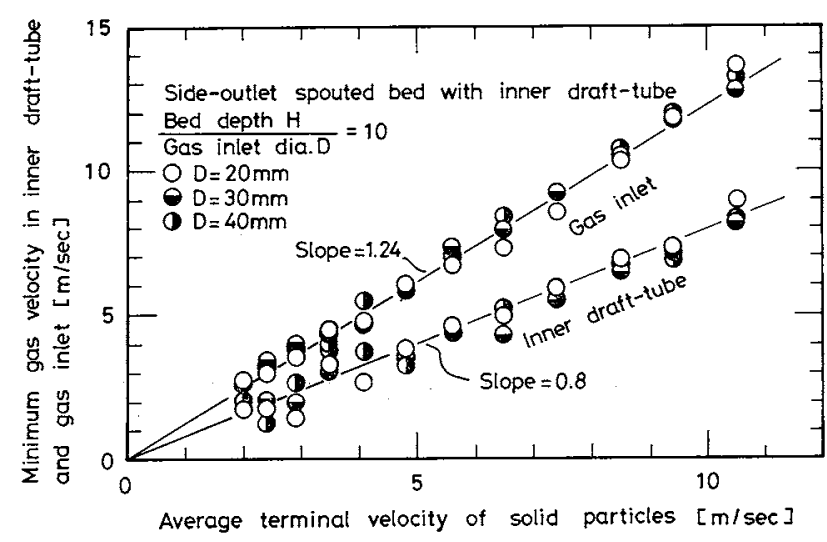

Fig. 4 Minimum gas velocities in inner draft-tube and in gas inlet of similar-shaped side-outlet spouted beds with inner draft-tube

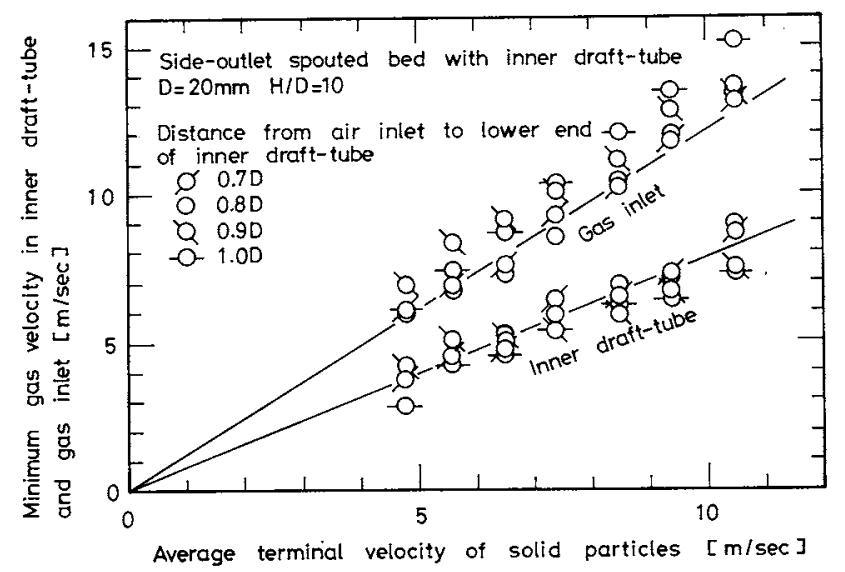

Fig. 5 Effect of distance from gas inlet to lower end of inner draft-tube on minimum gas velocities in inner draft-tube and in gas inlet

flow rate of gas which enters the apparatus is constant. Therefore, the ratio of the gas velocity in the inner draft-tube to the gas velocity in the gas inlet is also constant.
The above-mentioned ratio is held constant even if the particle size changes, as can be seen from Fig. 4. This is based on the fact that the shapes of gas streamlines are also similar even if the particle size changes. As far as we investigated, the shapes of gas streamlines were approximately similar in these cases and were not very different from the case shown in Fig. 3.

In the side-outlet spouted bed with inner drafttube which we used to investigate the gas velocity in the draft-tube or gas inlet, the distance from gas inlet to lower end of inner draft-tube was 0.8 time the diameter of gas inlet, as shown in Figs. 2 and 3. When the distance is changed, the void fraction in the inner draft-tube seems to be changed, and therefore the minimum gas velocity in the inner draft-tube changes. Figure 5 shows the minimum gas velocities both in the inner draft-tube and in the gas inlet for various distances from gas inlet to the lower end of the inner draft-tube. The two straight lines shown in Fig. 5 are quite the same as those shown in Fig. 4. In Fig. 5, deviations of the experimental data from the straight lines are found but are small.

\section{Applications to Scale-Up}

\section{1 Prediction of gas conversion}

In the case of side-outlet spouted bed with inner draft-tube, stable spouting sets in if the average gas velocity in the inner draft-tube becomes about 80 percent of the terminal velocity of solid particles, as mentioned above. Furthermore, for geometrically similar equipment, it has been found that the ratio of minimum gas velocity in gas inlet to terminal velocity of solid particles is unchanged even if the size of the apparatus or the size of particles changes. The latter characteristic is based on the fact that the shapes of gas 
streamlines are not changed even if the size of the apparatus or the size of particles changes. We tested only a few examples of geometrically similar spouted beds. However, if the same results can be obtained over a wide variety of conditions, the procedure for scale-up becomes quite simple, and the various performance characteristics such as conversion of gas or minimum spoutable flow rate of gas can easily be predicted.

Assuming that our results can be applied over a wide range of conditions, we have attempted to predict the increase in gas conversion of first-order solid-catalyzed reaction which accompanies the scale-up of the apparatus. The method of calculating gas conversion based on the observed gas streamlines in the annulus was discussed in detail in the previous paper ${ }^{3}$. To calculate the gas conversion by this method, a picture of the gas streamlines must be drawn. In the case of side-outlet spouted bed with inner draft-tube, however, the gas streamilines need not be determined for each reactor size and particle size, because the shape of gas streamlines becomes similar independently of reactor size and particle size. In such a case only one example of gas streamlines is needed. We used the gas streamlines obtained by use of the apparatus shown in Fig. 3 (A) and solid particles of $0.91 \mathrm{~mm}$ diameter.

In this way, gas conversions have been obtained for the various-sized side-outlet spouted beds of geometrically similar shapes shown in Fig. 3. The results are shown in Fig. 6. Thus, the size of reactor required to achieve a given conversion can be obtained.

Figure 7 illustrates the relation between minimum gas velocity in the gas inlet and terminal velocity of solid particles for two commonly used top-outlet spouted beds of geometrically similar shapes. The ratio of bed depth to diameter of gas inlet $H / D=7.0$. The size ratio of the two spouted beds is $1: 2$. No simple relation can be found between minimum gas velocity and terminal velocity of solid particles. So the procedure for scale-up is not easy in the case of the commonly used top-outlet spouted bed.

\section{2 Comparison of calculated gas conversions with} those of top-outlet spouted bed of Mathur et al.

We have again used the advantageous characteristic of the side-outlet spouted bed with inner drafttube, that is, the possibility of easily estimating gas conversion which accompanies scale-up. The gas conversion has been estimated for the side-outlet spouted bed with inner draft-tube of the same size as the top-outlet one of Mathur et al. As proposed in the previous paper ${ }^{1}$, Mathur and Lim estimated the gas conversion of first-order solid-catalyzed reaction for the top-outlet spouted bed, assuming the use of idealized porous catalyst and non-porous catalyst ${ }^{2}$. However, we cannot directly compare the gas conver-

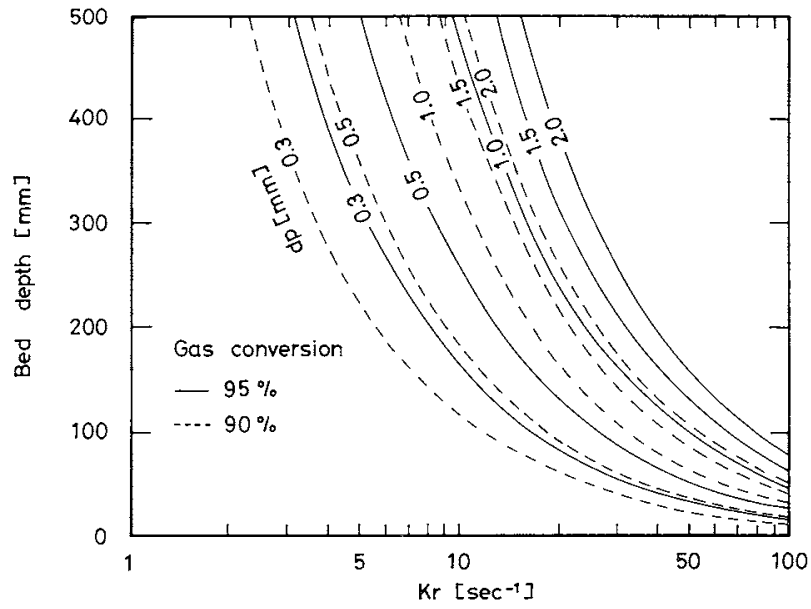

Fig. 6 Reactor size required for obtaining 90 and 95 percent gas coversion

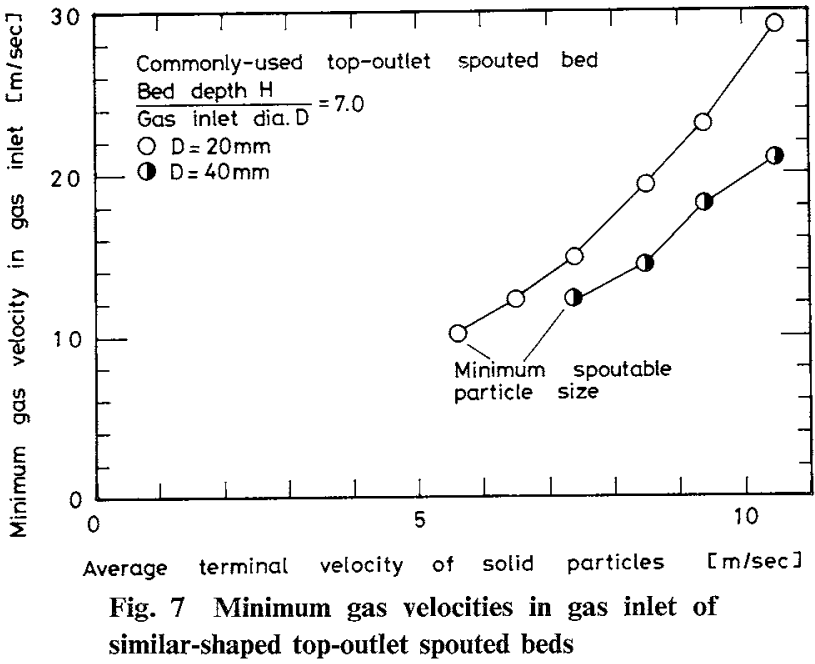

sions with Mathur and Lim's ones, because the size of the top-outlet spouted bed used by Mathur and Lim is much larger than that of our side-outlet spouted bed. Comparison of the gas conversion can be made by taking advantage of the characteristic of the sideoutlet spouted bed with inner draft-tube that gas conversion can be easily predicted when scale-up is carried out.

As mentioned above, the ratios of the minimum gas velocities in the inner draft-tube and in the gas inlet to the terminal velocity of solid particles are always constant for side-outlet spouted beds with inner drafttube of geometrically similar shapes. For spouted beds which are not geometrically similar, the ratios of both gas velocities to the terminal velocity of solid particles are not constant, but they are not very different, as shown in Figs. 2 and 5. Thus calculated conversion of gas is not greatly affected. Therefore, we used the relations shown in Fig. 4, assuming that they were applicable to a side-outlet spouted bed with inner draft-tube of the same size as Mathur and Lim's top-outlet one, as shown in Table 2. Furthermore, 


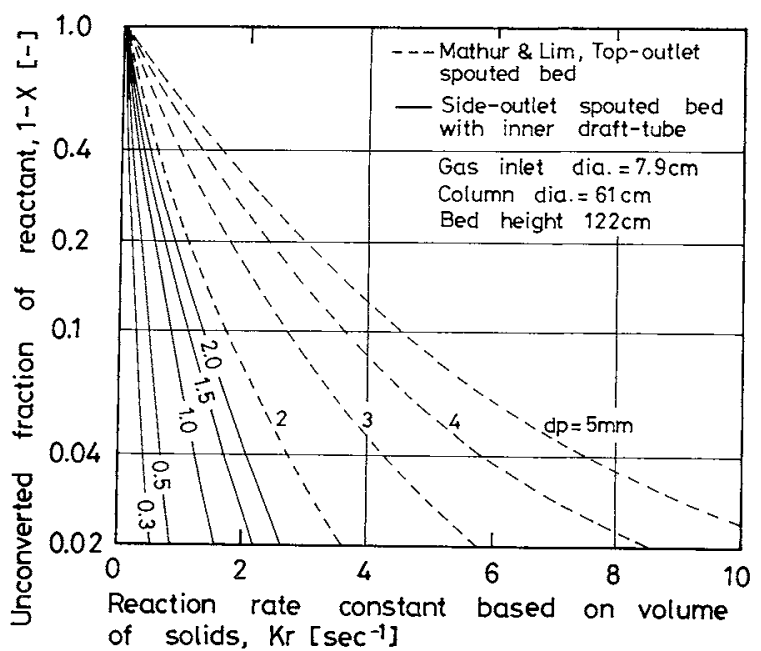

(Porous catalyst) (Reaction rate is independent of particle size.)

Fig. 8 Comparison of gas conversions with those of Mathur and Lim

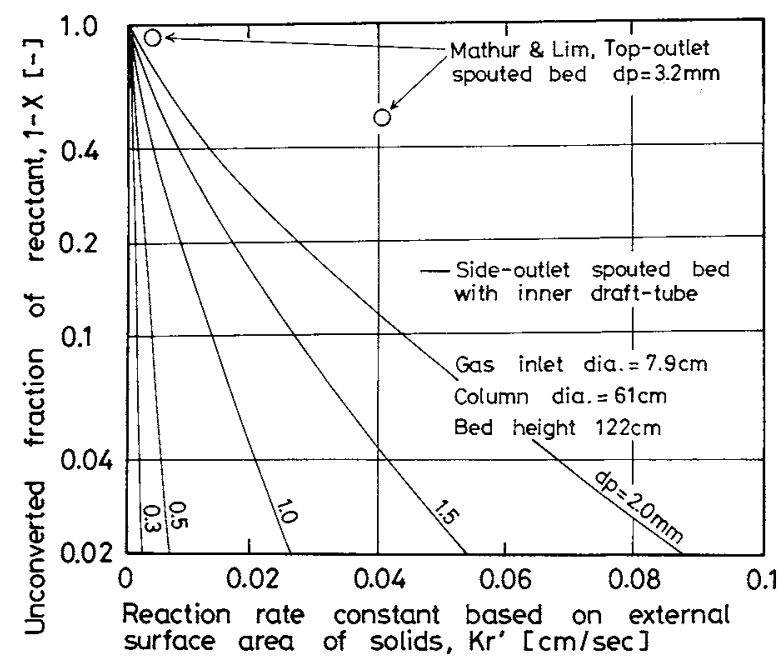

(Non-porous catalyst) (Reaction rate depends on particle size.)

Fig. 9 Comparison of gas conversions with those of Mathur and Lim

we assumed the specific gravity of solid particles as 1.4, following the example of Mathur and Lim. The terminal velocities of these particles are also shown in Table 2. Though the diameter of gas inlet was not specified in Mathur and Lim's paper ${ }^{2}$, we assumed it was the same as the diameter of the spout. The diameter of the spout is usually equal to the diameter of the gas inlet.

Calculated gas conversions for both the side-outlet spouted bed with inner draft-tube and Mathur and Lim's top-outlet spouted bed are shown in Figs. 8 and 9. Following Mathur and Lim's example, unconverted fraction of gas is plotted as ordinate and rate constant of first-order reaction as abscissa.

\section{Conclusion}

In the side-outlet spouted bed with inner draft-
Table 2 Reactor size and terminal velocity of solid particles

Mathur and Lim, Side-outlet Commonly used spouted bed with top-outlet spouted inner draft-tube bed

\begin{tabular}{lcc}
\hline $\begin{array}{l}\text { Column dia. } \\
\text { Bed depth }\end{array}$ & $61 \mathrm{~cm}$ & $61 \mathrm{~cm}$ \\
Spout dia. & $122 \mathrm{~cm}$ & $122 \mathrm{~cm}$ \\
Gas inlet dia. & $7.9 \mathrm{~cm}$ & \\
(=Inner draft-tube dia.) & $7.9 \mathrm{~cm}$ \\
Terminal velocity of solid particles \\
(Specific gravity $=1.4)$ \\
Average diameter & Terminal velocity \\
[mm] & [m/sec] \\
\hline 0.3 & 1.5 \\
0.5 & 2.6 \\
1.0 & 4.9 \\
1.5 & 6.8 \\
2.0 & 8.3 \\
\hline
\end{tabular}

tube, stable spouting sets in when the average gas velocity in the inner draft-tube becomes about 80 percent of the terminal velocity of solid particles. Furthermore, for geometrically similar apparatus, it has been found that the ratio of minimum gas velocity in the gas inlet to terminal velocity of solid particles is unchanged even if the size of the apparatus or the size of particles changes. The shapes of gas streamlines are kept similar when the size of the apparatus is enlarged with geometrically similarity.

It is apparent from the examples of calculating gas conversion that the above-mentioned characteristics of the side-outlet spouted bed with inner drafttube facilitate the procedure for scale-up.

\section{Acknowledgment}

We would like to express our thanks to Dr. Takashi Shirai of Tokyo Science University for his helpful suggestions. We are grateful to Dr. Taiichi Shibuya of Shinshu Unibersity for his helpful discussions and for reading the manuscript.

\section{Nomenclature}

\begin{tabular}{|c|c|}
\hline$D$ & $=$ gas inlet diameter \\
\hline$d_{p}$ & $=$ particle diameter \\
\hline$H$ & $=$ bed depth \\
\hline$K_{r}$ & $\begin{aligned}= & \text { reaction rate constant based on } \\
& \text { volume of solids }\end{aligned}$ \\
\hline$K_{r}^{\prime}$ & $\begin{aligned}= & \text { reaction rate constant based on } \\
& \text { external surface area of solids }\end{aligned}$ \\
\hline$U_{t}$ & $=$ terminal velocity of solid particles \\
\hline$X$ & $\begin{aligned}= & \text { fractional conversion of reactants } \\
& \text { leaving the reactor }\end{aligned}$ \\
\hline
\end{tabular}

\section{Literature Cited}

1) Hattori, H. and K. Takeda: J. Chem. Eng. Japan, 11, 125 (1978).

2) Mathur, K. B. and C. J. Lim: Chem. Eng. Sci., 29, 789 (1974).

3) Takeda, K. and H. Hattori: Kagaku Kogaku Ronbunshu, 2, 507 (1976).

(Presented at 46th Annual Meeting of The Soc. of Chem. Engrs., Japan, at Nagoya, April 3, 1981.) 